\title{
Anomalously Slow Cross Symmetry Phase Relaxation, Thermalized Non-Equilibrated Matter and Quantum Computing Beyond the Quantum Chaos Border
}

\author{
M. BIENERT ${ }^{\dagger}$, J. FLORES ${ }^{\dagger}$, S.YU. KUN ${ }^{\dagger, \ddagger, \S}$ and T.H. SELIGMAN ${ }^{\dagger}$ \\ $\dagger$ Centro de Ciencias Físicas, Universidad Nacional Autónoma de México, \\ Cuernavaca, Morelos, Mexico \\ E-mail: bienert@fis.unam.mx,jfv@servidor.unam.mx,kun@fis.unam.mx,seligman@fis.unam.mx \\ $\ddagger$ Nonlinear Physics Centre, RSPhysSE, ANU, Canberra ACT 0200, Australia \\ $\S$ Department of Theoretical Physics, RSPhysSE, ANU, Canberra ACT 0200, Australia
}

Received November 30, 2005, in final form February 08, 2006; Published online February 27, 2006

Original article is available at http://www.emis.de/journals/SIGMA/2006/Paper027/

\begin{abstract}
Thermalization in highly excited quantum many-body system does not necessarily mean a complete memory loss of the way the system was formed. This effect may pave a way for a quantum computing, with a large number of qubits $n \simeq 100-1000$, far beyond the quantum chaos border. One of the manifestations of such a thermalized nonequilibrated matter is revealed by a strong asymmetry around $90^{\circ} \mathrm{c} . \mathrm{m}$. of evaporating proton yield in the $\operatorname{Bi}(\gamma, \mathrm{p})$ photonuclear reaction. The effect is described in terms of anomalously slow cross symmetry phase relaxation in highly excited quantum many-body systems with exponentially large Hilbert space dimensions. In the above reaction this phase relaxation is about eight orders of magnitude slower than energy relaxation (thermalization).
\end{abstract}

Key words: anomalously slow cross symmetry phase relaxation; $\operatorname{Bi}(\gamma, \mathrm{p})$ photonuclear compound reaction; quantum chaos; thermalized non-equilibrated matter; quantum computing

2000 Mathematics Subject Classification: 82C10; 81V35; 81Q50; 34L25

\section{Introduction}

Independent-particle modes in interacting many-body systems result from a mean-field approximation and are at the center of many theoretical considerations. In the case of a quantum information device this independent-particle basis can be considered as the basis spanned by the individual qubits, the "computational basis". At high excitation energy, the interaction between the particles results in a rapid mixing of the independent particle states $[1,2,3,4]$. This mixing leads to the formation of complicated many-body configurations. Each of these individually ergodic (independent of the initial conditions) many-body states is characterized by sharing the energy between many particles of the system. The characteristic time for the formation of such thermalized many-body states is given by the inverse spreading width, $\tau_{\text {th }}=\hbar / \Gamma_{\text {spr }}[3,4]$. The quantity $\Gamma_{\text {spr }}$ also characterizes the width of the distribution of the expansion coefficients of the many-body eigenstates over a noninteracting mean-field basis $[1,2,3,4]$.

There is another way to interpret spreading width if there exists a classical analog to the system. Then it is given by the width of the energy distribution of trajectories determined by the Hamiltonian with interaction, measured in terms of the independent particle Hamiltonian. This distribution also provides a description of semiclassical wave functions [5] around which the actual wave functions have Gaussian fluctuations [6]. 
The question now arises whether phase relations and/or correlations between these individually ergodic, spatially extended, many-body states in the superposition may still preserve a memory of the way the system was excited. This question is of fundamental importance for the study of relaxation phenomena in nuclear, atomic, molecular and mesoscopic many-body systems, and for many-qubit quantum computation. In particular, we recently proposed [7] that, if phase relaxation is slower than energy relaxation, this can extend the time for quantum computing beyond the so called "quantum-chaos border" [8, 9].

To answer this question from first principles is presently not possible due to computational limitations. Indeed, in order to solve the full quantum many-body problem one would require a many-qubit quantum computer. Therefore, the only currently available resort to search for possible manifestations of long phase relaxation is the experiment, and a careful data analysis. Nuclear systems are an ideal testing ground to study many-body systems, since nuclear interactions are so strong that external perturbations can often be neglected. In particular, the analysis in $[7,10]$ of the data displaying forward peaking in angular distributions of evaporation protons from heavy nuclei in nucleon-induced reactions indicates that phase relaxation times can be up to five orders of magnitude longer than energy relaxation times.

In this paper we analyze another reaction that indicates the formation of thermalized nonequilibrated matter. This new form of matter was introduced by one of us [11, 12]. Again this is revealed by a strong asymmetry around $90^{\circ} \mathrm{c} . \mathrm{m}$. of the evaporating proton yield, but now in the $\operatorname{Bi}(\gamma, \mathrm{p})$ photonuclear reaction [13]. The experiment indicates that in this case the effect is even more pronounced. We shall see that here the phase relaxation is about eight orders of magnitude slower than thermalization, making the possibility of quantum computation beyond the quantum chaos border an even more attractive concept.

The present work is a step toward a more realistic situation because the entrance channel is given by electro-magnetic interaction. This is more similar to loading mechanisms in proposed quantum computers.

The present work does not suggest to use photonuclear reactions as a practical experimental setup for universal quantum computation. Indeed, one can not perform a universal set of gates for the considered photonuclear reaction. However the proposed analysis does demonstrate that phase relaxation can be much longer than thermalization which, in turn, illustrates the main idea of quantum computing far beyond the quantum chaos border.

\section{Experimental manifestation of thermalized non-equilibrated matter in $\operatorname{Bi}(\gamma, p)$ evaporation process}

We analyze the proton yield of the $\operatorname{Bi}(\gamma, \mathrm{p})$ photonuclear reaction produced by $24-\mathrm{MeV}$ bremsstrahlung. In Fig. 1 we present an angle-integrated photo-proton spectrum scaled with the outgoing proton energy $\varepsilon$ times the cross section $\sigma_{\text {inv }}(\varepsilon)$ of the inverse process of the capture of the proton with energy $\varepsilon$ by the residual nucleus. This $\varepsilon$-dependent inverse cross section is determined by the penetrability of the Coulomb and centrifugal barriers and was taken from [14] for a nuclear radius of $1.5 \times 10^{-13} A^{1 / 3} \mathrm{~cm}$, where $A$ is the nuclear mass number.

One can see that, for $\varepsilon \leq 8 \mathrm{MeV}$, the scaled spectrum has an exponential shape with a slope of $0.55 \mathrm{MeV}$. This is characteristic for the decay of thermalized compound nucleus with a "temperature" $T=0.55 \mathrm{MeV}$ of the residual nucleus. We evaluate the average excitation energy of the compound nucleus to be $\bar{E}^{*}=14 \mathrm{MeV}$, i.e. slightly above the center of the giant resonance peak at $13.5 \mathrm{MeV}$ [15]. For $\varepsilon=4 \mathrm{MeV}$, the average excitation energy of the residual nucleus is $\bar{E}_{\text {res }}^{*}=6.3 \mathrm{MeV}$. The average number of excitons (particles plus holes) [16] in the residual nucleus is $\bar{n}=\left(2 g \bar{E}_{\text {res }}^{*}\right)^{1 / 2} \simeq 14$, where $g=A / 13 \mathrm{MeV}^{-1}$ is the level density of independent particle states. The standard deviation of $n$ is about $(\bar{n} / 2)^{1 / 2} \simeq 2.6$. Therefore, the energy per 


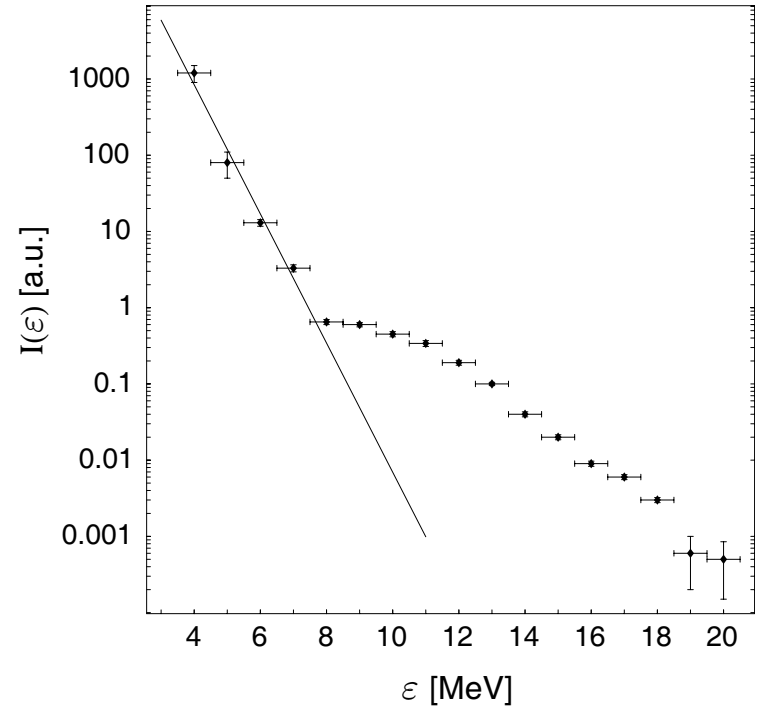

Figure 1. Scaled experimental angle-integrated spectrum (in arbitrary units) of protons of the $\operatorname{Bi}(\gamma, \mathrm{p})$ photonuclear reaction produced by $24 \mathrm{MeV}$ bremsstrahlung [13]. The line is exponential fit of the scaled spectrum for $\varepsilon \leq 8 \mathrm{MeV}$ with the slope (nuclear "temperature") of 0.55 $\mathrm{MeV}$ (see text).

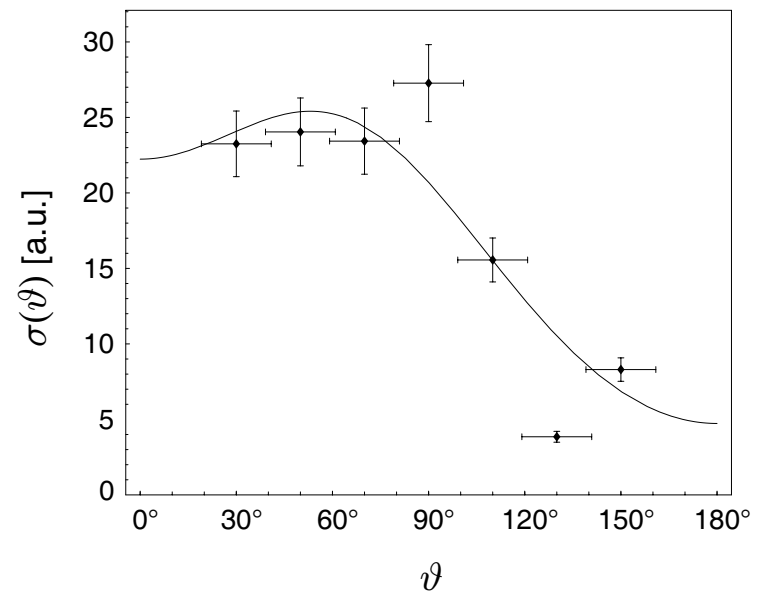

Figure 2. Experimental proton angular distributions (in arbitrary units) from the $\operatorname{Bi}(\gamma, \mathrm{p})$ photonuclear reaction for $\varepsilon=2-8 \mathrm{MeV}$ [13]. The curve is a fit to the experimental data (see text).

exciton in the residual nucleus, i.e. the nuclear "temperature", is $T=0.38-0.55 \mathrm{MeV}$. This is consistent with the fit in Fig. 1 for $\varepsilon \leq 8 \mathrm{MeV}$ with $T=0.55 \mathrm{MeV}$. Similarly we find that the temperature of the $\mathrm{Bi}$ compound nucleus with the excitation energy $14 \mathrm{MeV}$ is $0.57-0.78 \mathrm{MeV}$ which is roughly consistent with the fit in Fig. 1. This implies thermalization of both compound and residual nuclei [14].

In Fig. 2 we present experimental proton angular distributions from the $\operatorname{Bi}(\gamma, p)$ photonuclear reaction for $\varepsilon=2-8 \mathrm{MeV}$ [13]. One can see that, in spite of a complete energy relaxation in the thermalized compound nucleus, the angular distributions are strongly asymmetric about $90^{\circ}$, i.e. memory of the direction of the incident $\gamma$-ray beam is clearly retained.

\section{Determination of the cross symmetry phase relaxation width}

In order to identify anomalously slow phase relaxation behind the forward peaking of the evaporating protons we make use of a standard formula for double differential cross section in $\gamma$-ray induced reactions [17]:

$$
\begin{aligned}
\sigma(\theta)= & \left(\lambda^{2} / 8 \pi\right)(2 I+1)^{-1} \sum S_{\alpha L_{1} p ; \alpha^{\prime} l_{1}^{\prime} s^{\prime}}^{J_{1} \pi_{1}}(E)^{*} S_{\alpha L_{2} p ; \alpha^{\prime} l_{2}^{\prime} s^{\prime}}^{J_{2} \pi_{2}}(E) \\
& \left(L_{1} 1-11 \mid L_{1} 1 l_{1} 0\right)\left(L_{2} 1-11 \mid L_{2} 1 l_{2} 0\right) \Delta\left(l_{1}, p\right) \Delta\left(l_{2}, p\right) \\
& {\left[\left(2 J_{1}+1\right)\left(2 J_{2}+1\right)\right]^{1 / 2} i^{L_{2}-L_{1}+l_{1}-l_{2}}(-1)^{s^{\prime}-I-L+L_{1}-L_{2}+1} W\left(J_{1} L_{1} J_{2} L_{2} ; L\right) } \\
& Z\left(l_{1} L_{1} l_{2} L_{2} ; 1 L\right) Z\left(l_{1}^{\prime} J_{1} l_{2}^{\prime} J_{2} ; s^{\prime} L\right) P_{L}(\theta),
\end{aligned}
$$

where the sum is over $L_{1}, L_{2}, l_{1}, l_{2}, J_{1}, J_{2}, \pi_{1}, \pi_{2}, l_{1}^{\prime}, l_{2}^{\prime}, s^{\prime}, p$ and $L$. Here $\lambda$ is the wave length of the electromagnetic radiation, $E$ the total energy of the system, $I$ the spin of the target nucleus, and $\alpha, \alpha^{\prime}$ are microstates of the reaction partners in the entrance and exit channels, accordingly. $J_{1}, J_{2}$ are the total spins of the compound nucleus, $\pi_{1}, \pi_{2}$ its parities, $L_{1}, L_{2}$ the total angular momenta of multipoles, $l_{1}, l_{2}$ the orbital momenta in the entrance and $l_{1}^{\prime}, l_{2}^{\prime}$ in the exit channel, 
and $s^{\prime}$ is the channel spin in the exit channel. The symbol $p$ is defined to have the value 0 for magnetic radiation and 1 for electric radiation. For $p=0, \Delta\left(l_{1(2)}, p\right)=\delta_{l_{1(2)}, L_{1(2)}}$ and, for $p=1, \Delta\left(l_{1(2)}, p\right)=\delta_{l_{1(2)}, L_{1(2)} \pm 1}$. In equation $(1),(\cdot \mid \cdot)$ denote the Clebsch-Gordan coefficients, $W$ are the Racah coefficients defined in [18], and the $Z$ coefficients are defined in [19]. $S$-matrix elements are in a mixed representation in which the entrance channel states are in the multipole representation, and the exit channel states are in the channel spin representation.

In what follows we use a symplified form of equation (1). We neglect the proton spin in the exit channel so that the spin of the residual nucleus, $I^{\prime}$, is equal to the exit channel spin. We neglect the target spin in the entrance channel, $I=0$. Therefore, $J_{1(2)}=L_{1(2)}$. We take into account the proton orbital angular momenta $l_{1,2}^{\prime} \leq 2$ only since, for $l_{1,2}^{\prime} \geq 3$, evaporating protons are significantly sub-barrier due to the centrifugal and Coulomb barriers. We consider electric dipole $\left(L_{1,2}=1\right)$ and quadrupole $\left(L_{1,2}=2\right)$ radiation only. As a result, equation (1) takes the form

$$
\begin{aligned}
\sigma(\theta)= & \left(\lambda^{2} / 8 \pi\right) \sum S_{\alpha L_{1} ; \alpha^{\prime} l_{1}^{\prime}}^{L_{1}}(E)^{*} S_{\alpha L_{2} ; \alpha^{\prime} l_{2}^{\prime}}^{L_{2}}(E) \\
& \left(L_{1} 1-11 \mid L_{1} 1 l_{1} 0\right)\left(L_{2} 1-11 \mid L_{2} 1 l_{2} 0\right) \delta_{l_{1}, L_{1} \pm 1} \delta_{l_{2}, L_{2} \pm 1} \\
& i^{L_{2}-L_{1}+l_{1}-l_{2}}(-1)^{I^{\prime}+1} Z\left(l_{1} L_{1} l_{2} L_{2} ; 1 L\right) Z\left(l_{1}^{\prime} L_{1} l_{2}^{\prime} L_{2} ; I^{\prime} L\right) P_{L}(\theta)
\end{aligned}
$$

where the sum is taken over $L_{1}, L_{2}, l_{1}, l_{2}, l_{1}^{\prime}, l_{2}^{\prime}, I^{\prime}$ and $L$. In equation (2), the states with different total spins, $L_{1} \neq L_{2}$, correspond to opposite parities. For $L_{1}=L_{2}\left(L_{1} \neq L_{2}\right)$, the sum in equation (2) includes the terms with even (odd) values of $\left|l_{1}^{\prime}-l_{2}^{\prime}\right|$ only.

The key element in a description of the asymmetry of the evaporating protons is a correlation between fluctuating $S$-matrix elements with different total spins $L_{1} \neq L_{2}\left(L_{1}=1\right.$ and $L_{2}=2$ or $L_{1}=2$ and $L_{2}=1$ ) and parities [11, 12]:

$$
\left\langle S_{\alpha L_{1} ; \alpha^{\prime} l_{1}^{\prime}}^{L_{1}}(E)^{*} S_{\alpha L_{2} ; \alpha^{\prime} l_{2}^{\prime}}^{L_{2}}(E)\right\rangle=\frac{\left[\left\langle\left|S_{\alpha L_{1} ; \alpha^{\prime} l_{1}^{\prime}}^{L_{1}}(E)\right|^{2}\right\rangle\left\langle\left|S_{\alpha L_{2} ; \alpha^{\prime} l_{2}^{\prime}}^{L_{2}}(E)\right|^{2}\right\rangle\right]^{1 / 2}}{\left(1+\beta / \Gamma_{\mathrm{cn}}\right)},
$$

where the brackets $\langle\cdot\rangle$ stand for ensemble averaging which is equivalent to the energy $(E)$ averaging under the ergodicity condition. The physical meaning of the spin and parity off-diagonal ("cross symmetry") phase relaxation width $\beta$, introduced in $[20,11,12]$, is the characteristic inverse time over which the interference between the states with different total spins and parities does not vanish upon the energy averaging and therefore the memory of the direction of the initial beam is preserved [11, 12]. If this phase memory time $\tau_{p h}=\hbar / \beta$ is about as long or longer than the average life time $\hbar / \Gamma_{\mathrm{cn}}$ of the compound nucleus then the evaporating protons are emitted asymmetrically about $90^{\circ}$ c.m., i.e. the memory about the direction of the initial beam is retained. However, if the phase memory time is much shorter than the average life time of the compound nucleus then the spin and parity off-diagonal correlations vanish, memory on the direction of the initial beam is lost leading to the conventional Bohr picture of compound nucleus with the symmetric about $90^{\circ} \mathrm{c.m}$. angular distributions. Therefore, deviation of the angular distributions of the evaporating particles from the symmetry around $90^{\circ} \mathrm{c.m}$. indicates that $\beta$ is smaller or comparable to $\Gamma_{\mathrm{cn}}$.

For the orbital momentum off-diagonal correlation between $S$-matrix elements with the same total spins and parities we have $[11,12]$

$$
\left\langle S_{\alpha L ; \alpha^{\prime} l_{1}^{\prime}}^{L}(E)^{*} S_{\alpha L ; \alpha^{\prime} l_{2}^{\prime}}^{L}(E)\right\rangle=\left[\left\langle\left|S_{\alpha L ; \alpha^{\prime} l_{1}^{\prime}}^{L}(E)\right|^{2}\right\rangle\left\langle\left|S_{\alpha L ; \alpha^{\prime} l_{2}^{\prime}}^{L}(E)\right|^{2}\right\rangle\right]^{1 / 2} .
$$

Both the equations (3) and (4) reflect a strong correlation between the partial width amplitudes and, as a result, between $S$-matrix elements with different orbital momenta $\left(l_{1}^{\prime} \neq l_{2}^{\prime}\right)$ of evaporating protons referred to $[11,12]$ as the continuum correlation. Note that such a strong 
correlation between reduced width amplitudes corresponding to the same total spin and parity values but different orbital momenta was experimentally revealed for a number of compound nuclei in the regime of isolated resonances [21].

The statistical model [14] yields

$$
\left\langle\left|S_{\alpha L ; \alpha^{\prime} l^{\prime}}^{L}(E)\right|^{2}\right\rangle=T_{\alpha}^{L} T_{\alpha^{\prime} l^{\prime}}^{L} / \sum_{\alpha^{\prime} l^{\prime}} T_{\alpha^{\prime} l^{\prime}}^{L}
$$

Here, $T_{\alpha}^{L} \equiv T^{L}$ are the entrance channel transmission coefficients for the formation of the compound nucleus with the total spins $L=1$ and $L=2$ due to the absorption of electric dipole and quadrupole radiation, accordingly. The exit channel transmission coefficients are assumed to be independent of the compound nucleus spin $L$ and the spin of the residual nucleus $I^{\prime}$ [14], $T_{\alpha^{\prime} l^{\prime}}^{L} \equiv T^{l^{\prime}}$.

We use equations (2), (3), (4) and (5) for the analysis the experimental angular distributions in Fig. 2. We find that, upon energy averaging and summation over microstates of the residual nucleus, apart from the overall normalization constant, a shape of the angular distributions depends on the four parameters: $A=T^{L=2} / T^{L=1}, B=T^{l^{\prime}=1} / T^{l^{\prime}=0}, C=T^{l^{\prime}=2} / T^{l^{\prime}=0}$, and $\beta / \Gamma_{\mathrm{cn}}$.

From the fit of the experimental angular distributions in Fig. 2 we obtain: $A=0.082$, $B=0.47, C=0.37$ and $\beta / \Gamma_{\mathrm{cn}}=0.11$. The compound nucleus' decay width $\Gamma_{\mathrm{cn}}$ for $\mathrm{Bi}$ with an excitation energy of $14 \mathrm{MeV}$ can be estimated from the systematics in Fig. 7 of [22], which provides a good description of the experimentally determined $\Gamma_{\mathrm{cn}}$ for a wide range of mass numbers. From this estimation we obtain $\Gamma_{\mathrm{cn}} \simeq 0.1 \mathrm{eV}$ what yields in turn $\beta \simeq 0.01 \mathrm{eV}$. At the same time, the standard nuclear physics estimate for the spreading width of Bi nucleus with the excitation energy $14 \mathrm{MeV}$ is about $2 \mathrm{MeV}$ (see Fig. 2.1 in [23]). This is close to another estimate of $\Gamma_{\mathrm{spr}}$ as a width of a dipole giant resonance [3], which is about $4.5 \mathrm{MeV}$ for $\mathrm{Bi}$ [15]. It corresponds to an exponentially large effective dimension of Hilbert space given by $N_{\text {eff }} \simeq$ $\Gamma_{\text {spr }} / D \simeq 10^{16}$, where $D \simeq 10^{-16} \mathrm{MeV}$ is the average level spacing of the Bi compound nucleus with the excitation energy $14 \mathrm{MeV}$. Note that the total spin and parity off-diagonal $S$-matrix correlations for evaporation processes were justified in [12] in the limit $N_{\text {eff }} \rightarrow \infty$. For the cross symmetry phase relaxation time much longer than the energy relaxation time, the formalism also leads to (i) quantum-classical transition [24, 25], stable coherent rotation [26, 27, 28], Schrödinger cat states $[29,30]$ in complex collisions in the regime of strongly overlapping resonances of the intermediate system, and (ii) spontaneous correlations, non-equilibrium phase transitions [31] and anomalous sensitivity in finite highly excited many-body systems [32].

It should be noted that while we have been able to determine an anomalously small value of $\beta$ from the data analysis, its theoretical evaluation is currently an open problem. Therefore more theoretical insight is needed for a deeper understanding of the effect of anomalously slow cross symmetry phase relaxation in highly excited quantum many-body systems.

\section{An illustration of the idea of quantum computing far beyond the quantum chaos boarder}

The idea of quantum computing on a time scale $\tau_{\mathrm{ph}}=\hbar / \beta \simeq 6 \times 10^{-14}$ sec., which is much longer than the thermalization time $\hbar / \Gamma_{\text {spr }} \simeq 3 \times 10^{-22}$ sec., can be illustrated as follows [7]. Consider the $\operatorname{Bi}(\gamma, \mathrm{p})$ compound nucleus reaction to be the quantum protocol. The single-particle basis is the quantum register. The entrance channel represents the loading process. The measurement of the angular distribution of the yield of evaporating protons plays the role of the readout. In accordance with the standard criterion [8,9], a quantum computer melts down at $t \geq \hbar / \Gamma_{\mathrm{spr}}$. If so, then any information about specific features of the loaded state (entrance channel) is lost 
while the protocol is executed. Yet, as was shown in the previous section, our "read out" (the angular distribution of evaporation protons) shows that our "protocol" yields non-trivial results eight orders of magnitude later than the time scale $\hbar / \Gamma_{\mathrm{spr}} \simeq 3 \times 10^{-22}$ sec. for the onset of quantum chaos in our "quantum computer". These results depend on the "loading", i.e. the direction of the incident beam.

In order to demonstrate that the obtained phase memory time $\tau_{\mathrm{ph}}=\hbar / \beta \simeq 6 \times 10^{-14}$ sec. is indeed a very long time scale of the system we note that during this time a nucleon at the Fermi energy crosses the Bi compound nucleus about $10^{8}$ times. Yet, this phase memory time is still about eight orders of magnitude shorter than the Heisenberg time $\hbar / D \simeq 10^{-5}$ sec., where $D \simeq 10^{-16} \mathrm{MeV}$ is the average level spacing of the Bi compound nucleus at an excitation energy of $14 \mathrm{MeV}$.

Clearly, the spectrum of the Bi compound nucleus in the above considered reaction is not resolved since $\Gamma_{\mathrm{cn}} / D \simeq 10^{9}$, i.e. resonances of the compound nucleus are strongly overlapping. Therefore, all exponentially large information hidden in the Hilbert space is not available. However we have demonstrated that useful information, such as the phase relaxation time, still can be obtained even though the quantum protocol time is about nine orders of magnitude shorter than the Heisenberg time for our "quantum computer". This is in accord with a similar observation in [9].

\section{Conclusions}

We have demonstrated that thermalization in highly excited quantum many-body system does not necessarily mean a complete loss of memory of the way the system was formed. Manifestation of such a thermalized non-equilibrated matter has been revealed from the analysis of a strong asymmetry around $90^{\circ} \mathrm{c} . \mathrm{m}$. of the evaporating proton yield in the $\operatorname{Bi}(\gamma, \mathrm{p})$ photonuclear reaction. We have shown that thermalized non-equilibrated matter can exist for time spans of $\sim 6 \times$ $10^{-14}$ sec., which is eight orders of magnitude longer than thermalization time in this example. This indicates that long lived transient states can exist in many-body systems with exponentially large dimensions of Hilbert space. If a quantum computer with a large number of qubits $n \simeq 100$ 1000 can be brought into such a state, this may provide a solution for the scaling problem which is one of the central challenges of quantum information [33].

\section{Acknowledgments}

S. Kun is grateful to the organizers of the 6th International Conference "Symmetry in Nonlinear Mathematical Physics", which took place in his beautiful home city Kyiv in June 2005, for the kind invitation and the opportunity to give a talk. We acknowledge financial support from PAPIIT project 10803 and CONACyT project 41000-F. M. Bienert is supported by a FeodorLynen fellowship of the Alexander-von-Humboldt foundation.

[1] Wigner E.P., Characteristic vectors of bordered matrices with infinite dimensions, Ann. Math., 1955, V.62, N 3, 548-564.

Wigner E.P., Characteristic vectors of bordered matrices with infinite dimensions II, Ann. Math., 1957, V.65, N 2, 203-207.

[2] Wigner E., Statistical properties of nuclei, Editor J.B. Garg, New York - London, Plenum Press, 1972 , p. 11.

[3] Anderson P.W., Basic notions of condensed matter physics, Frontiers in Physics, Vol. 55, The BenjaminCummings, 1984, 71-72.

[4] Guhr T., Müller-Groeling A., Weidenmüller H.A., Random matrix theories in quantum physics: common concepts, Phys. Rep., 1998, V.299, N 4-6, 189-425 (and references therein); cond-mat/9707301.

[5] Benet L., Izrailev F.M., Seligman T.H., Suárez-Moreno A., Semiclassical properties of eigenfunctions and occupation number distribution for a model of two interacting particles, Phys. Lett. A, 2000, V.277, N 2, 87-93; chao-dyn/9912035. 
[6] Benet L., Flores J., Hernandez-Saldana H., Izrailev F.M., Leyvraz F., Seligman T.H., Fluctuations of wavefunctions about their classical average J. Phys. A: Math. Gen., 2003, V.36, N 5, 1289-1297; nlin.CD/0207039.

[7] Flores J., Kun S.Yu., Seligman T.H., Slow phase relaxation as a route to quantum computing beyond the quantum chaos border, Phys. Rev. E, 2005, V.72, N 1, 017201, 4 pages.

[8] Georgeot B., Shepelyansky D.L., Quantum chaos border for quantum computing, Phys. Rev. E, 2000, V.62, N 3, 3504-3507; quant-ph/9909074.

[9] Shepelyansky D.L., Quantum chaos and quantum computers, in Proceedings of Nobel Symposium on Quantum Chaos 2000, Phys. Scripta, 2001, V.90, 112-120; quant-ph/0006073.

[10] Bienert M., Flores J., Kun S.Yu., Experimental proposal for accurate determination of the phase relaxation time in highly excited quantum many-body systems, nucl-ex/0508020.

[11] Kun S.Yu., Novel approach to angular distributions in precompound reactions: Does the Bohr hypothesis always work?, Z. Phys. A, 1994, V.348, N 2, 273-279.

[12] Kun S.Yu., Statistical reactions with memory and thermalized-nonequilibrated nuclear states, Z. Phys. A, 1997, V.357, N 2, 255-269.

[13] Toms M.E., Stephens W.E., Photoprotons from In, Ce, and Bi, Phys. Rev., 1953, V.92, N 2, $362-366$.

[14] Blatt J.M., Weisskopf V.F., Theoretical nuclear physics, New York, Dover Publications, Inc., 1991.

[15] Harvey R.R., Caldwell J.T., Bramblett R.L., Fultz S.C., Photoneutron cross sections of $\mathrm{Pb}^{206}, \mathrm{~Pb}^{207}, \mathrm{~Pb}^{208}$, and $\mathrm{Bi}^{209}$, Phys. Rev., 1964, V.136, N 1, B126-B131.

[16] Blann M., Preequilibrium decay, Annu. Rev. Nucl. Sci., 1975, V.25, 123-166.

[17] Simon A., Theory of polarized particles and gamma rays in nuclear reactions, Phys. Rev., 1953, V.92, N 4, 1050-1060.

[18] Racah G., Theory of complex spectra. I, Phys. Rev., 1942, V.61, N 3-4, 186-197. Racah G., Theory of complex spectra. II, Phys. Rev., 1942, V.62, N 9-10, 438-462.

[19] Blatt J.M., Biedenharn L.C., The angular distributions of scattering and reaction cross sections, Rev. Mod. Phys., 1952, V.24, N 4, 258-272.

[20] Kun S.Yu., Ericson fluctuations for the finite spin relaxation times, Phys. Lett. B, 1993, V.319, N 1, 16-22.

[21] Mitchell G.E., Bilpuch E.G., Shriner J.F., Lane A.M., Amplitude correlations in nuclear resonance spectroscopy, Phys. Rep., 1985, V.117, N 1, 1-74.

[22] Ericson T., Mayer-Kuckuk, Fluctuations in nuclear reactions, Annu. Rev. Nucl. Sci., 1966, V.16, $183-206$.

[23] Agassi D., Weidenmüller H.A., Mantzouranis G., The statistical theory of nuclear reactions for strongly overlapping resonances as a theory of transport phenomena, Phys. Rep., 1975, V.22, N 3, 145-179.

[24] Kun S.Yu., Vagov A.V., Greiner W., Quantum-classical correspondence in microscopic and mesoscopic complex collisions, Phys. Rev. C, 2001, V.63, N 1, 014608, 5 pages.

[25] Benet L., Kun S.Yu., Wang Qi, Effect of phase relaxation on quantum superpositions in complex collisions, quant-ph/0503046.

[26] Kun S.Yu., Vagov A.V., Vorov O.K., Coherently rotating hyperdeformed quasimolecules in ${ }^{12} \mathrm{C}+{ }^{24} \mathrm{Mg}$ scattering?, Phys. Rev. C, 1999, V.59, N 2, R585-R588.

[27] Kun S.Yu., Robson B.A., Vagov A.V., Oscillating-correlated nonstatistical structures, slow spin decoherence, and hyperdeformed coherent rotational states in ${ }^{24} \mathrm{Mg}+{ }^{24} \mathrm{Mg}$ and ${ }^{28} \mathrm{Si}+{ }^{28} \mathrm{Si}$ scattering, Phys. Rev. Lett., 1999, V.83, N 3, 504-507.

[28] Kun S.Yu., Chadderton L.T., Vagov A.V., Greiner W., A new probe for coherent many-body dynamics: Nonergodic molecules in continuum, Int. J. Mod. Phys. E, 2002, V.11, N 4, 273-280.

[29] Kun S.Yu., Benet L., Chadderton L.T., Greiner W., Haas F., Macroscopic quantum superpositions in highly excited strongly interacting many-body systems, Phys. Rev. C, 2003, V.67, N 1, 011604, 4 pages; quant$\mathrm{ph} / 0205036$.

[30] Benet L., Kun S.Yu., Wang Qi, Denisov V., Effect of a finite-time resolution on Schrödinger cat states in complex collisions, Phys. Lett. B, 2005, V.605, N 1-2, 101-105; nucl-th/0407029.

[31] Kun S.Yu., Spontaneous coherence and non-equilibrium correlation phase transitions in microscopic and mesoscopic systems, in Proceedings of International Conference "Nonequilibrium and Nonlinear Dynamics in Nuclear and Other Finite Systems" (May 21-25, 2001, Beijing), Editors Zhuxia Li, Ke Wu, Xizhen Wu, Enguang Zhao and F. Sakata, AIP Conference Proceedings, Vol. 597, New York, Melville, 2001, 319-326.

[32] Kun S.Yu., Sensitivity of nucleus-nucleus cross sections and atomic-electron effects in dissipative heavy-ion collisions, Phys. Rev. Lett., 2000, V.84, N 3, 423-426.

[33] Nielsen M.A., Chuang I.L., Quantum computation and quantum information, Oxford, Cambridge University Press, 2000. 